\title{
Fluctuating Position-Related Cognitive Disturbances and Recurrent Cerebral Ischemic Attacks as Presenting Symptoms in a Patient with Platypnea-Orthodeoxia Syndrome
}

Carlo Lovati ${ }^{*}$, Massimo Suardelli and Pierluigi Bertora

Neurology Unit, Headache Center, Osp. L. Sacco, University of Milan, Via G.B. Grassi 74, 20157, Milan, Italy

"Corresponding author: Carlo Lovati, Neurology Unit, Headache Center, Osp. L. Sacco, University of Milan, Via G.B. Grassi 74, 20157, Milan, Italy, Tel: +390239042317; E-mail: carlo.lovati@asst-fbf-sacco.it

Received Date: Nov 29, 2017; Accepted Date: Dec 26, 2017; Published Date: Jan 06, 2018

Copyright: $\odot 2018$ Lovati C, et al. This is an open-access article distributed under the terms of the Creative Commons Attribution License, which permits unrestricted use, distribution, and reproduction in any medium, provided the original author and source are credited.

\begin{abstract}
Platypnea-orthodeoxia syndrome is a condition of dyspnea and hypoxia whilst in the upright position, which improves in the recumbent position. We present a case of platypnea-orthodeoxia due to a fenestrated atrial septal aneurysm that induced recurrent strokes and a recent condition of fluctuating confusion and cognitive impairment, modified by position, associated with rapid variations of $\mathrm{O}_{2}$ saturation position related. The suspect of platypneaorthodeoxia syndrome may be hypothesized in case of patients with recurrent cerebral ischemia and fluctuating cognitive disturbances induced by change of position. In those cases a careful echocardiographic evaluation and $\mathrm{O}_{2}$ saturation determination in up and downright position are required.
\end{abstract}

Keywords: Platypnea-orthodeoxia syndrome; Dementia

\section{Background}

Platypnea-orthodeoxia syndrome is a rare condition in which dyspnea and hypoxia concurrently appear in the upright position and are relieved by clinostatism. Pulmonary and right atrial pressures are not elevated [1]. Platypnea-orthodeoxia (POS) could be explained on the basis of positional modification of abnormal shunting. Some conditions are associated with this rare syndrome, including cardiac, pulmonary or, in a few cases, hepatic alterations [2], which can account for the variable concomitant clinical manifestations in these patients.

Although hypoxemia is likely to induce central nervous system impairment; neurological abnormalities have been reported in an exceptionally low number of cases. In few reported cases POS induced recurrent neurological manifestations as falls and confusion [3]. We present a patient with typical manifestations of POS in whom the appearance of neurological symptoms brought him to medical attention.

\section{Case Report}

A 82-year-old male was admitted to hospital for the acute appearance of cognitive disturbances and reduction of consciousness with a fluctuating evolution. Past medical history included hypertension, a thoracic intervention at the age of 76 for hydropneumothorax, polidistrectual vasculopathy and four symptomatic cerebral ischemic episodes in different districts as confirmed by cerebral computed tomography. A brother of the patient had died for heart disease (ventricular aneurysm of unknown origin). The cardiorespiratory examination was normal. The electrocardiogram showed sinus tachycardia (105 bpm) with left atrial enlargement and incomplete right bundle branch block. Arterial blood gas analysis showed mild hypoxemia ( $\mathrm{pH}$ 7.47, $\mathrm{PaCO}_{2} 25 \mathrm{mmHg}, \mathrm{PaO}_{2} 63 \mathrm{mmHg}$,
$\mathrm{O}_{2}$ saturation 93\%). CBC showed mild hypochromatic microcytic anemia. During the hospital stay the patient presented acute left superoexternal quadrantanopsia and neglect. Fluctuating hypoxia was reported, which was unmodified by $\mathrm{O}_{2}$ administration but relieved in the recumbent position. Position dependence of hypoxia was documented by pulse oxymetry. While breathing room air $\mathrm{SpO} 2$ was consistently above $90 \%$ in the supine position but constantly dropped to as low as $80 \%$ when sitting together with the appearance of worsening dyspnea and cyanosis. No clinical, laboratory or radiographic signs of acute heart failure, cardiac ischemia, peripheral venous thrombosis or pulmonary thromboembolism were found.

Transthoracic (TTE) and transesophageal echocardiogram (TEE) showed the presence of an aneurysmal interatrial septum. Color Doppler flow measurement demonstrated the presence of an atrial septal defect (ASD) with unusually abundant fenestrations. Right ventricular contractile function was normal. TEE was performed both in the supine and sitting position. Significant right-to-left shunting, while absent in recumbent position, appeared at a 70 degree incline. The aortic root was mildly dilated with distortion of the interatrial septum. A Swan Ganz catheterism confirmed normal pulmonary artery pressures.

It was decided to proceed to the endovascular repair of the atrial septal defect (details). The particular anatomic conditions requested the positioning of multiple devices in order to obtain a satisfactory reduction of the shunting.

\section{Discussion}

In our patient both clinical and laboratory findings suggested an unusual disorder known as platynea-orthodeoxia syndrome. This pathological condition has been described in association to pulmonary diseases - including pulmonary arteriovenous malformations, chronic obstructive bronchopneumopathy, pulmonary fibrosis, kyphoscoliosis - or cardiac diseases in presence of secundum atrial septal defect or patent foramen ovale - particularly in association with septal 
Citation: Lovati C, Suardelli M and Bertora P (2018) Fluctuating Position-Related Cognitive Disturbances and Recurrent Cerebral Ischemic Attacks as Presenting Symptoms in a Patient with Platypnea-Orthodeoxia Syndrome. Med Rep Case Stud 3: 147. doi: $10.4172 / 2572-5130.1000147$

Page 2 of 2

interatrial aneurysm, aortic abnormalities, right hydrothorax, tricuspidal defect, eosinophilic endocarditis, after thoracotomy or pneumectomy. Hepatopulmonary syndrome was even reported as possible cause of this syndrome. Echocardiographic investigation allowed us to identify the presence of a large atrial septal defet with Right to Left shunting which appeared only in upright position. This abnormality can therefore be considered as the responsible for POS in our patient.

Right-to-left shunting is hard to explain in the absence of elevated right atrial pressure. Nonetheless, some mechanisms have been suggested to cause this abnormality, including compression of the right atrium in the upright posture, decreased compliance of the right ventricle and anatomic anomalies in the vena cava insertion to right atrium and atrial septum [1].

Besides the large fenestrated atrial septal aneurysm, our patient also presented a distortion of the large vessels which was more evident in orthostatism. We raised the hypothesis that - whilst in the upright position - an abnormally oriented insertion of the superior vena cava could generate a blood "jet" directly towards the thinned septum, thereby enhancing bulging during atrial systole and leftward flow through the defect.

In all the similar cases we have analyzed in literature, only respiratory and cardiac symptoms occurred at onset. Overt neurological pictures were rarely described. Focal neurological signs are a plausible consequence of localized cerebral infarction caused by paradoxical embolism through a patent foramen ovale or any other interatrial septal defect [4]. Position-related dyspnea however is the only rule you can consider to suspect POS.

It is hard to define why neurological symptoms were preminent in our case. Probably old age, sideropenic anemia, polidistrectual vasculopathy contributed to fluctuating cognitive disturbances and to hemodynamic strokes in watershed fields. Moreover, embolic stroke remains a constant risk for patient with atrial enlargement and a huge interatrial septal defect. Neuroimaging in our patient seems to confirm both these hypotheses.

\section{Conclusions}

A high index of suspicion is required to suspect this rare syndrome. Whereas POS usually manifests itself with position-related respiratory symptoms (mainly dyspnea), neurological manifestations are rarely reported. The extreme variability of the clinical pictures needs a multidisciplinary approach. Our patient is unique since neurologic manifestations consistent with cerebral hypoxia (altered consciousness and cognitive disturbance) and changing according to the position caught the clinician's attention. The consequences of POS on cerebral function should therefore carefully evaluated in patients with this syndrome, especially when possible therapeutic interventions are prospected. If it is already mandatory to evaluate patients with multiple recurrent cryptogenic strokes with transesophageal echocardiogram, it is advisable to consider echocardiogram and dynamic pulse oxymetry when fluctuating cognitive disturbances or consciousness disorders are the only alarms.

\section{References}

1. Van Gaal WJ, Joseph M, Jones E, Matalanis G, Horrigan M (2005) Platypnea-orthodeoxia associated with a fenestrated atrial septal aneurysm: case report. Cardiovasc Ultrasound 13: 3-28.

2. Patel AD, Abo-Auda WS, Nekkanti R, Ahmed S, Razmi RM, et al. (2003) Platypnea-orthodeoxia in a patient with ostium primum atrial septal defect with normal right heart pressures. Echocardiography 20(3): 299-303.

3. Putot A, Laborde C, Fichot M, Brunel P, Deidda M, et al. (2017) Falls and Delirium: Platypnea-Orthodeoxia Syndrome. Am J Med pii: S0002-9343(17)31183-X.

4. Ohara T, Nakatani S, Hashimoto S, Akaiwa Y, Yazaki S, et al. (2007) A case of platypnea-orthodeoxia syndrome in a patient with a pulmonary arteriovenous fistula and a patent foramen ovale. J Am Soc Echocardiogr 20(4): 439. 